\title{
Perfil clínico-epidemiológico de pacientes do Ambulatório de Alergia Ocular da Santa Casa de São Paulo
}

\author{
Clinical and epidemiological profileof patients diagnosed and treated at the Ocular \\ Allergy Sector at Santa Casa de São Paulo
}

\author{
Denise Atique Goulart ${ }^{1}$ \\ Dario Grechi Goulart ${ }^{2}$ \\ Marcela Colussi Cypel ${ }^{3}$ \\ Paulo Elias Correa Dantas ${ }^{4}$ \\ Maria Cristina Nishiwaki-Dantas 5
}

\begin{tabular}{|c|}
\hline RESUMO \\
\hline 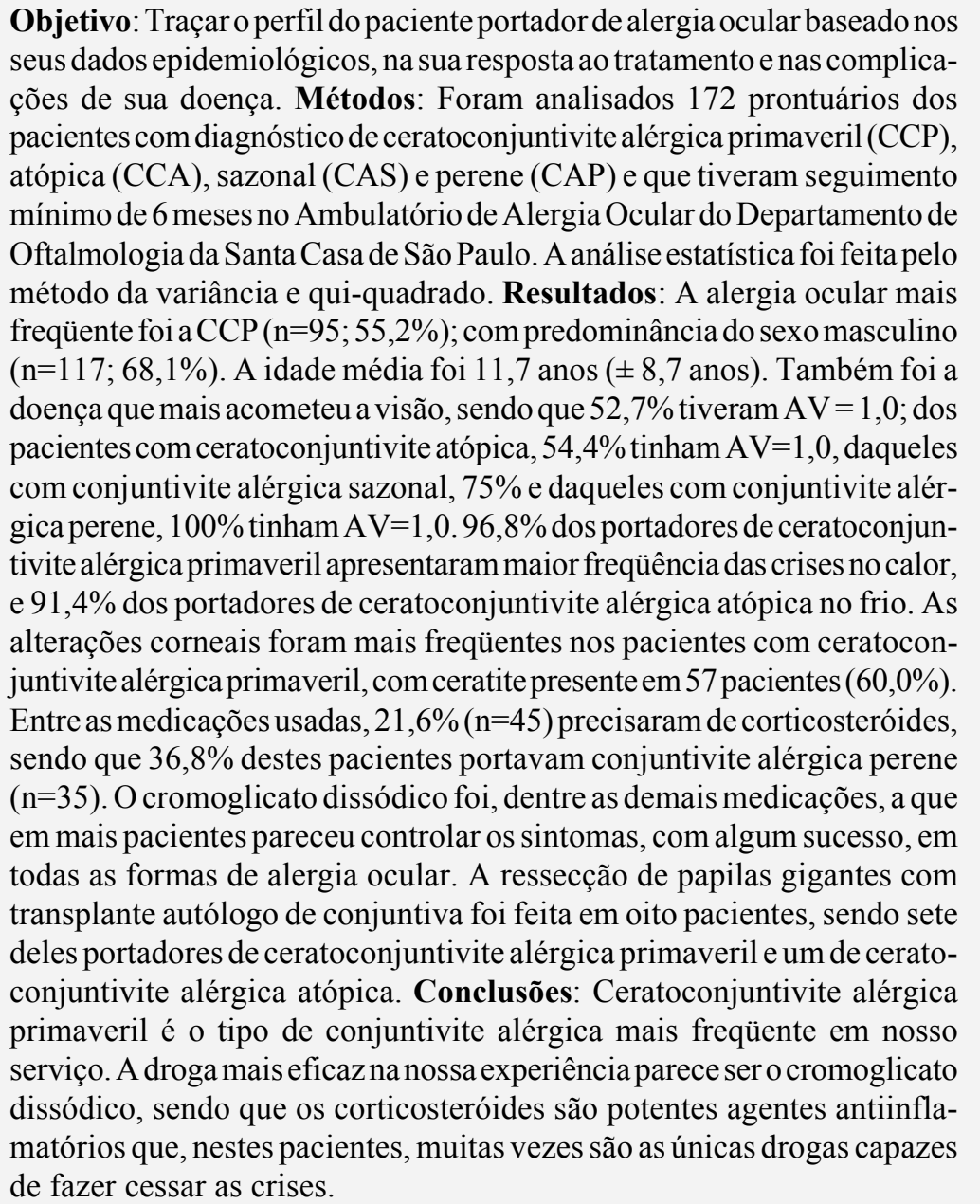 \\
\hline
\end{tabular}

Descritores: Conjuntivite alérgica/epidemiologia; Conjuntivite alérgica/complicações; Conjuntivite alérgica/quimioterapia; Ceratoconjuntivite/quimioterapia; Cromoglicato dissódico/uso terapêutico; Cromoglicato dissódico/administração \& dosagem
${ }^{1}$ Médica Assistente do Setor de Córnea e Moléstias Externas do Departamento de Oftalmologia da Santa Casa de São Paulo, Pós-graduanda, nível doutorado, pela Universidade Federal de São Paulo - UNIFESP. 2 Médico Assistente do Setor de Retina e Vítreo do Departamento de Oftalmologia da Santa Casa de São Paulo, Pós-graduando, nível doutorado, pela Universidade de São Paulo - USP

${ }^{3}$ Médica Curso de Aperfeiçoamento do Pronto-Socorro do Departamento de Oftalmologia da Santa Casa de São Paulo.

${ }^{4}$ MédicoAssistente do Setor de Córnea e Moléstias Externas do Departamento de Oftalmologia da Santa Casa de São Paulo, Médico Chefe do Setor de Córnea do Hospital Oftalmológico de Sorocaba, Doutor em Medicina pela Universidade de São Paulo - USP.

${ }^{5}$ Médica Chefe do Setor de Córnea e Moléstias Externas e de Alergia do Departamento de Oftalmologia da Santa Casa de São Paulo, Doutora em Medicina pela Universidade Federal de São Paulo - UNIFESP.

Endereço para correspondência: Denise Atique Goulart, Av. Prof. Múcio Lobo da Costa 82, casa 4 - Jundiaí - (SP) CEP 13208-710 E-mail: dagoulart@uol.com.br

Recebido para publicação em 28.06.2002 Aceito para publicação em 08.01.2003

\section{INTRODUÇÃOO}

O termo atopia é usado para descrever a reação alérgica em indivíduos com predisposição genética a doenças que demonstrem positividade na reação de Prausnitz-Kustner. A existência de atopia, encontrada em 15\% da 
população geral, indica doença alérgica e predisposição a doenças como as conjuntivites alérgicas ${ }^{(1)}$.

As conjuntivites alérgicas cursam com quadro clínico característico de prurido, fotofobia, lacrimejamento, hiperemia, podendo ocorrer perdas visuais permanentes devido ao uso indevido de corticosteróides tópicos e às alterações corneais em manifestações graves de ceratoconjuntivite atópica e primaveril. É importante que o diagnóstico do quadro alérgico ocular e do subtipo de conjuntivite seja feito com precisão o mais precocemente possível, a fim de se instituir rapidamente o tratamento adequado e se evitar complicações ${ }^{(2)}$.

As mais comuns formas de conjuntivite alérgica são a sazonal e a perene, geralmente associadas à rinite ou asma brônquica. São também as formas mais brandas de alergia ocular, o que faz com que, muitas vezes, estes pacientes não procurem o oftalmologista ${ }^{(1)}$.

O quadro clínico da conjuntivite alérgica sazonal caracteriza-se por episódios sazonais de prurido, hiperemia conjuntival discreta a moderada e hipertrofia papilar da conjuntiva palpebral superior com papilas menores que $1 \mathrm{~mm}$. Raramente há envolvimento corneal ${ }^{(3)}$.

A forma conjuntivite alérgica perene (CAP) é semelhante à conjuntivite alérgica sazonal (CAS), porém com sintomas menos severos e ausência de sazonalidade. Em ambas, a patogênese se dá por reação de hipersensibilidade tipo I. Nos exames laboratoriais, encontra-se presença de eosinófilos ou grânulos eosinofílicos no raspado conjuntival, embora sua ausência não descarte a possibilidade de alergia ocular ${ }^{(3)}$.

A dermatoceratoconjuntivite atópica (CCA) é um quadro inflamatório crônico que afeta bilateralmente conjuntivas e córneas em 15 a $40 \%$ dos indivíduos com dermatite atópica. De crises freqüentes no inverno, tem predileção pacientes do sexo masculino entre e segunda e a quinta década de vida ${ }^{(4)}$. Trata- se de reação de hipersensibilidade tipo I e IV e o quadro clínico apresenta, além do prurido intenso, lacrimejamento, secreção mucosa, hiperemia, visão embaçada, fotofobia e dor. Os sintomas estão presentes o ano todo, mas se exacerbam no frio e na presença de certos alérgenos ${ }^{(3)}$. Sinais importantes são a hiperplasia gelatinosa do limbo, pontos de HornerTrantas e hipertrofia papilar mais proeminente inferiormente. Freqüentemente há formação de cicatrizes conjuntivais com perda de células caliciformes que podem resultar em olho seco secundário. Também pode cursar com ceratite punctata superficial, ceratocone e neovascularização periférica ${ }^{(1,3)}$.

$\mathrm{O}$ raspado conjuntival na CCA mostra menor número de eosinófilos, geralmente menos degranulados.

A ceratoconjuntivite primaveril (CCP) é também afecção inflamatória bilateral, porém autolimitada, tendendo a se resolver espontaneamente na puberdade. Mais freqüente em meninos, tem exacerbações sazonais, sendo mais comum na primavera e verão, e em regiões de clima quente e $\operatorname{seco}^{(3,5)}$.

Duplo mecanismo de hipersensibilidade tipo I e IV parece estar envolvido na gênese da CCP, cujo quadro clínico pode se apresentar de duas formas: palpebral e limbar ${ }^{(4,6)}$. A forma palpebral se apresenta com hipertrofia papilar superior maior que $1 \mathrm{~mm}$; casos severos cursam com perda dos septos e confluência das papilas, formando as papilas gigantes. A forma limbar se dá com reação papilar no limbo, que assume aspecto gelatinoso e espessado, com possível presença de pontos de Horner-Trantas, que são compostos por eosinófilos e indicam atividade da doença ${ }^{(4)}$.

$\mathrm{Na}$ CCP é comum haver comprometimento corneal, inicialmente com ceratite punctata causada pelos mediadores liberados pela conjuntiva e exacerbada pelo trauma mecânico das papilas gigantes sobre a córnea. Pode haver coalescência destes pontos resultando em um defeito epitelial conhecido como úlcera em escudo. Esta se mostra oval, horizontal, superior e com depósito de placa de fibrina superficialmente ${ }^{(6-7)}$. No raspado conjuntival, há presença de eosinófilos e principalmente grânulos eosinofílicos ${ }^{(3)}$.

Todas as formas de alergia têm tratamento direcionado para a identificação do alérgeno e medidas gerais, como eliminar cortinas, tapetes, forrar cobertores de lã, na tentativa de evitar contato com os principais alérgenos que são o pó, pólen e os pêlos de animais.

Como as manifestações sazonais e perenes tendem a ser autolimitados, só se institui tratamento medicamentoso quando houver sinais e sintomas realmente importantes.

Compressas geladas para alívio do prurido, em casos leves. Lágrimas artificiais sem preservantes diluem e ajudam a drenar o alérgeno e mediadores inflamatórios presentes na superfície ocular. Vasoconstritores tópicos, como a fenilefrina reduzem a hiperemia conjuntival na fase aguda. Podem ser usados até 5 vezes ao dia, por até 7 dias $^{(8)}$.

Anti-histamínicos tópicos, como a levocabastina $0,05 \%$ e a emedastina, bloqueiam reversivelmente os receptores de histamina localizados primariamente nas pálpebras e conjuntivas, prevenindo, assim, a ocupação e estimulação dos mesmos pelas moléculas de histamina. O uso oral destes medicamentos não é sugerido, devido aos efeitos colaterais e à necessidade de altas doses para atingir a concentração ocular necessária. A via tópica tem efeito imediato e não acarreta efeitos adversos.

A levocabastina $0,05 \%$ é bloqueador $\mathrm{H}_{1}$ que mostra diminuição dos sintomas em até 4 horas após a instilação. Estudos mostram que a emedastina reduz significativamente a produção de citocinas ${ }^{(1,3,9)}$.

Pacientes com as formas perene e sazonal raramente necessitam de estabilizador da membrana de mastócito ou corticoterapia.

$\mathrm{Na}$ forma atópica, o tratamento se mostra inicialmente como nas formas sazonal e perene, porém é comum a necessidade de estabilizadores de membrana de mastócito (lodoxamida $0,1 \%$ e cromoglicato de sódio $4 \%$ ) e até mesmo corticoterapia para haver controle dos sintomas.

Estabilizadores de membrana de mastócitos atuam impedindo o afluxo de cálcio para a membrana celular dos mastócitos, mas também inibem a ativação de neutrófilos, eosinófilos e monócitos. Além disso, provou- se que são eficazes em inibir diretamente a ativação de leucócitos, bloqueando a destruição tecidual 
normalmente causada pelas células inflamatórias. Estas drogas também parecem inibir a liberação de substância $\mathrm{P}$ e outros neuropeptídeos a partir de terminações nervosas ${ }^{(1)}$.

Existem também as medicações de ações combinadas, como o cetotifeno e a olopatadina; o primeiro atua como antihistamínico, estabilizador de membrana de mastócito e inibe a migração de eosinófilos, enquanto a olopatadina não tem ação sobre os eosinófilos. Estes medicamentos são uma boa alternativa para pacientes que precisam fazer uso da medicação cronicamente $^{(4)}$.

Antiinflamatórios não hormonais, como o ceterolac de trometamina $0,5 \%$ podem ser benéficos para casos moderados e em conjuntivites sazonais e perenes. Sua ação é na inibição da ação da ciclooxigenase ${ }^{(1)}$. Estudos mostram melhora do prurido e da inflamação ${ }^{(2)}$.

Casos severos necessitam terapêutica com corticosteróides tópicos potentes, como fosfato de dexametasona $0,1 \%$ ou acetato de prednisolona 1\%, em altas doses (a cada 3 horas), por curto período de tempo (até 7 dias). ${ }^{2}$ Estas drogas agem inibindo a fosfolipase $\mathrm{A}_{2}$. A queda na produção de mediadores inflamatórios resulta em diminuição da permeabilidade capilar e exsudação celular, bloqueio do influxo de leucócitos para a conjuntiva e liberação de enzimas hidrolíticas pelas células inflamatórias.

Não se pode deixar de lembrar que a corticoterapia local pode causar glaucoma, catarata, predisposição a infecções e perfuração ocular ${ }^{(1)}$.

O tratamento da CCP é igual ao da CCA na maioria dos casos. Deve-se lembrar que pacientes com úlcera em escudo necessitam tratamento com corticóides tópicos em altas doses por curto período de tempo e as placas de fibrina devem ser removidas. Casos de CCP relativamente controlada, porém com lesões corneais decorrentes das papilas gigantes devem ser submetidos à ressecção das papilas com transplante autólogo de conjuntiva ${ }^{(10-11)}$.

Este estudo foi realizado com objetivo de traçar um perfil do portador das diferentes formas de alergia ocular, com dados epidemiológicos e tentar delinear cada uma destas doenças. Também procurou-se relatar as respostas destes pacientes às diferentes medicações utilizadas para seu tratamento, bem como as principais complicações.

\section{MÉTODOS}

Foram analisados os prontuários de 172 pacientes do ambulatório de alergia ocular do Departamento de Oftalmologia da Santa Casa de Misericórdia de São Paulo, no período de janeiro de 1996 a fevereiro de $2001 \mathrm{em}$ estudo retrospectivo e descritivo.

Foram incluídos neste estudo somente aqueles pacientes que tiveram tempo de seguimento mínimo de 6 meses.

Os pacientes foram classificados quanto a sexo, idade, tipo de conjuntivite alérgica, acuidade visual no melhor olho (com a correção óptica), alterações corneais mais encontradas, medicações usadas, resposta ao tratamento, número de medica- ções usadas até o controle da doença, uso de corticosteróide tópico, tempo de uso da medicação por crise, período do ano em que mais crises ocorreram e necessidade de ressecção de papilas gigantes.

Os dados foram analisados estatisticamente pelo método de variância e qui-quadrado. Apenas o grupo com diagnóstico de conjuntivite alérgica perene não pôde ser estatisticamente analisado devido ao pequeno tamanho da amostra.

\section{RESULTADOS}

A ceratoconjuntivite primaveril foi a alergia ocular mais freqüente em nosso ambulatório, com 95 pacientes $(55,2 \%)$, seguida pela dermatoceratoconjuntivite atópica, com $35(20,3 \%)$, pela conjuntivite alérgica sazonal, com $32(18,6 \%)$ e pela conjuntivite alérgica perene com 10 pacientes (5,9\%). (Gráfico 1)

A idade variou de 1 a 48 anos, com média de 11,4 anos e desvio padrão de 8,6 anos. Para CAS a média de idade dos pacientes foi $13,4 \pm 10,3$ anos (variou de 3 a 48 anos), para CCA foi de $15,6 \pm 12,1$ anos (variou de 3 a 46 anos) e para CCP foi 9, $1 \pm 5,01$ anos ( 2 a 23 anos). CAP de 16,5 anos $\pm 10,1$ anos, variando de 8 a 43 anos. As diferenças entre as idades foram estatisticamente significantes ( $p<0,001$ e $F=9,08)$. (Tabela1)

Quanto à acuidade visual destes pacientes, no melhor olho com correção óptica, a CCP mostrou 50 pacientes (52,7\%) com $\mathrm{AV}=1,0$; enquanto para CCA este número foi de 19 pacientes $(54,4 \%)$, para CAS foi de 24 (75\%) e CCP, 10 pacientes (100\%). (Tabela 2)

$\mathrm{Na}$ CCP as crises foram mais freqüentes no calor $(\mathrm{n}=92$; $96,8 \%)$, e na CCA, no frio ( $n=32 ; 91,4 \%)$. A CAS mostrou- se pouco mais freqüente no calor ( $\mathrm{n}=23 ; 71,9 \%)$ e a CAP 2 pacientes referiram ter sintomas o ano todo. A análise estatística mostrou $\mathrm{p}<0.001$ para CAS, CCA e CCP. (Gráfico II)

Quanto ao sexo, o masculino correspondeu a 117 pacientes $(68,1 \%)$ e o feminino, $55(31,9 \%)$. O sexo masculino foi predominante nos 4 tipos de alergia ocular estudados. Na CCP, 64 dos 95 pacientes eram meninos $(67,4 \%)$; na CCA eles correspondiam a $60,0 \%(\mathrm{n}=21)$; na CAS $81,2 \%(\mathrm{n}=26)$ e na CAP, $60,0 \%(n=6)$. Não houve diferença estatisticamente significativa entre os grupos com CAS, CCA e CCP $(p>0,16)$.

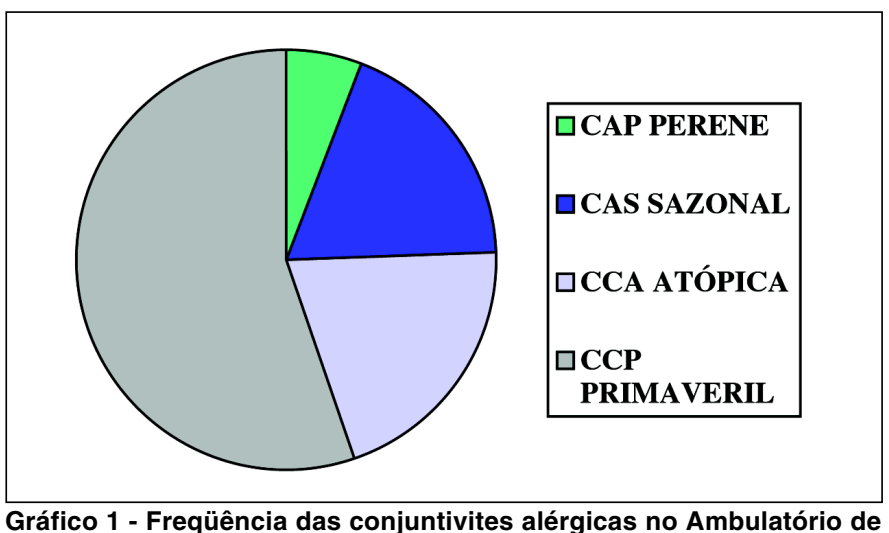

Gráfico 1 - Freqüência das conjuntivites alérgicas no Ambulatório de Alergia Ocular da Santa Casa de Misericórdia de São Paulo 


\begin{tabular}{|c|c|c|c|c|}
\hline Conjuntivite & $\begin{array}{l}\text { Idade média } \\
(95 \%) \text { máximo }\end{array}$ & $\begin{array}{l}\text { Desvio padrão } \\
(95 \%) \text { mínimo }\end{array}$ & Intervalo de confiança & Intervalo de confiança \\
\hline Sazonal & 13,4 & 10,3 & 17,1 & 9,7 \\
\hline Primaveril & 9,1 & 5,0 & 10,2 & 8,1 \\
\hline Atópica & 15,6 & 12,1 & 19,7 & 11,4 \\
\hline Total & 11,4 & 8,5 & 12,7 & 10,0 \\
\hline
\end{tabular}

\begin{tabular}{|c|c|c|c|c|}
\hline AV & CAP & CAS & CCA & CCP \\
\hline $\mathrm{NI}$ & 0 & $5(15,7 \%)$ & $4(11,6 \%)$ & $16(16,8 \%)$ \\
\hline 1,0 & $10(100 \%)$ & $24(75,0 \%)$ & $19(54,4 \%)$ & $50(52,7 \%)$ \\
\hline 0,9 & 0 & $1(3,1 \%)$ & 0 & $3(3,1 \%)$ \\
\hline 0,8 & 0 & 0 & $1(2,8 \%)$ & $3(3,1 \%)$ \\
\hline 0,7 & 0 & $1(3,1 \%)$ & $1(2,8 \%)$ & $4(4,3 \%)$ \\
\hline 0,6 & 0 & 0 & $1(2,8 \%)$ & $2(2,1 \%)$ \\
\hline 0,5 & 0 & 0 & $2(5,7 \%)$ & $1(1,0 \%)$ \\
\hline 0,4 & 0 & 0 & $2(5,7 \%)$ & $5(5,2 \%)$ \\
\hline 0,3 & 0 & 0 & 0 & $4(4,3 \%)$ \\
\hline 0,2 & 0 & 0 & $1(2,8 \%)$ & $2(2,1 \%)$ \\
\hline 0,1 & 0 & $1(3,1 \%)$ & $1(2,8 \%)$ & $4(4,3 \%)$ \\
\hline$<0,1$ & 0 & 0 & $3(8,6 \%)$ & $1(1,0 \%)$ \\
\hline
\end{tabular}

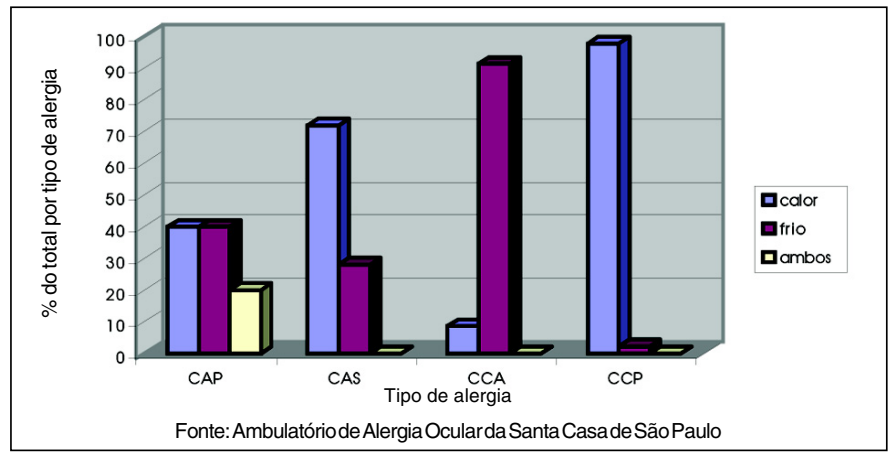

Gráfico II - Distribuição das alergias de acordo com o clima

Sessenta e seis pacientes $(38,4 \%)$ não apresentaram alterações corneais, 17 pacientes $(9,9 \%)$ tiveram úlcera em escudo, $77(44,8 \%)$ ceratite e 12 apresentaram ceratocone (7,0\%). Estas manifestações foram mais freqüentes na $\mathrm{CCP}$, na qual 57 pacientes $(60,0 \%)$ tiveram ceratite, 14 , úlcera em escudo $(14,7 \%)$ e 6, ceratocone $(6,4 \%)$. (Tabela 3 )

Quanto às medicações usadas para o controle do quadro alérgico, como lágrimas artificiais, anti-histamínicos estabilizadores de membrana dos mastócitos e medicações combinadas, analisamos cada tipo de alergia separadamente.

Entre os pacientes com CCP, 50,5\% controlaram o quadro clínico com a primeira medicação usada $(n=48)$. As drogas

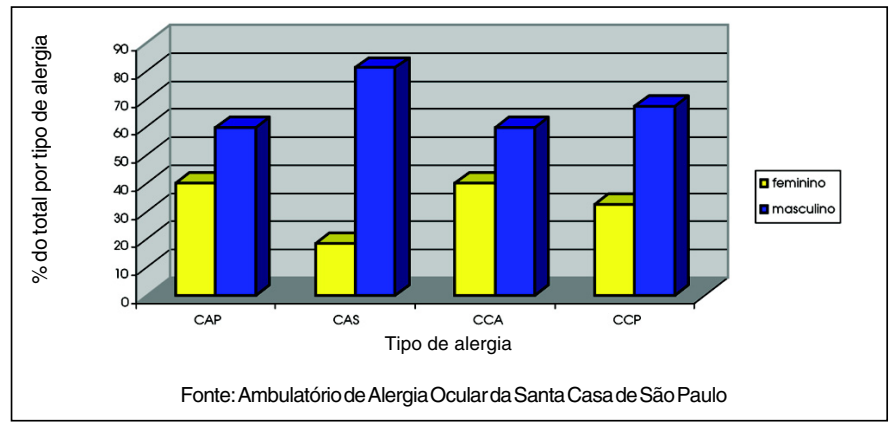

Gráfico III - Distribuição dos pacientes quanto ao sexo

\begin{tabular}{|c|c|c|c|c|}
\hline & $\begin{array}{c}\text { CAP } \\
(\mathrm{N}=10)\end{array}$ & $\begin{array}{c}\text { CAS } \\
(\mathrm{N}=32)\end{array}$ & $\begin{array}{c}\text { CCA } \\
(\mathrm{N}=35)\end{array}$ & $\begin{array}{c}\text { CCP } \\
(\mathrm{N}=95)\end{array}$ \\
\hline Ceratite & 0 & $7(21,8 \%)$ & $13(37,2 \%)$ & $57(60,0 \%)$ \\
\hline úlcera em escudo & 0 & 0 & $3(8,6 \%)$ & $14(14,7 \%)$ \\
\hline Ceratocone & 0 & $1(3,1 \%)$ & $5(14,2 \%)$ & $6(6,4 \%)$ \\
\hline
\end{tabular}

mais usadas no tratamento foram o cromoglicato dissódico $4 \%$, usado em 35 dos 95 pacientes $(36,8 \%)$ e a lodoxamida $0,1 \%$, em 37 pacientes $(39,0 \%)$, esta última controlou o quadro definitivamente para a maioria dos pacientes. (Tabela 4) 


\begin{tabular}{|c|c|c|c|c|}
\hline Droga de controle & CCP & CCA & CAS & CAP \\
\hline Lágrima & $5(5,2 \%)$ & 0 & $6(18,7 \%)$ & $1(10 \%)$ \\
\hline Levocabastina & $2(2,2 \%)$ & $2(5,7 \%)$ & $3(9,4 \%)$ & 0 \\
\hline Emedastina & $1(1,0 \%)$ & $1(2,8 \%)$ & $2(6,2 \%)$ & 0 \\
\hline Cromoglicato & $35(36,8 \%)$ & $16(45,8 \%)$ & $9(28,1 \%)$ & $6(60 \%)$ \\
\hline Lodoxamida & $37 \quad(39,0 \%)$ & $11(31,4 \%)$ & $8(25,0 \%)$ & $3(30 \%)$ \\
\hline Olopatadina & $10(10,5 \%)$ & $4(11,5 \%)$ & $3(9,4 \%)$ & 0 \\
\hline Cetotifeno & $3(3,1 \%)$ & $1(2,8 \%)$ & 0 & 0 \\
\hline Keterolac & $2(2,2 \%)$ & 0 & $1(3,1 \%)$ & 0 \\
\hline Total & $95(100 \%)$ & $35(100 \%)$ & $32(100 \%)$ & $10(100 \%)$ \\
\hline
\end{tabular}

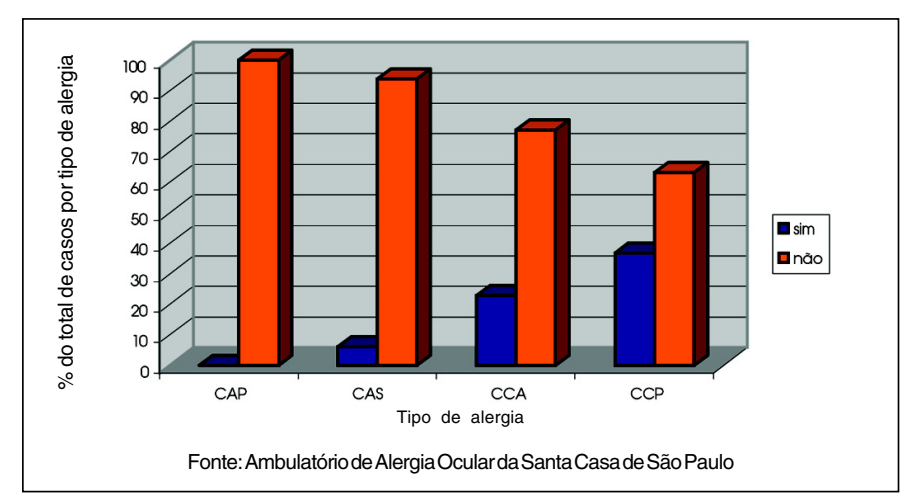

Gráfico 4 - Distribuição do uso de corticóides por tipo de alergia

Para CCA, 57,1\% dos pacientes controlaram a alergia com a primeira medicação $(n=20)$; a primeira escolha foi cromoglicato dissódico em 14 pacientes (40\%), droga esta que também foi a mais eficaz tanto no controle definitivo $(n=16 ; 45,8 \%)$ como no controle do quadro clínico na primeira tentativa $(n=12 ; 60 \%)$.

Os pacientes com diagnóstico de conjuntivite alérgica sazonal também se beneficiaram mais com o cromoglicato dissódico $4 \%$, que foi a droga de primeira escolha em $28,1 \%$ dos pacientes $(n=9)$. Este também foi o número de pacientes que conseguiram controle do quadro clínico com esta medicação. A droga que mais controlou a CAS como primeira medicação foi também o cromoglicato, em $28,5 \%(n=6)$.

Dos dez pacientes com diagnóstico de conjuntivite alérgica perene, 8 controlaram o quadro com a primeira droga usada $(80 \%)$, sendo que $62,5 \%$ deles usaram cromoglicato dissódico $4 \%$ e os $37,5 \%$ restantes, lodoxamida $(n=3)$. A droga que mais controlou definitivamente o quadro de alergia ocular nestes pacientes foi também o cromoglicato, com 6 pacientes $(60,0 \%)$.

Dos pacientes com CCP, $50,5 \%(n=48)$ controlaram a alergia com a primeira medicação introduzida; na CCA, 57,1\% $(n=20)$; na CAS, $86,6 \%(n=21)$ e na CAP, $80 \%(n=8)$.

Sete pacientes com $\mathrm{CCP}(7,4 \%)$ foram submetidos à ressecção de papilas gigantes com transplante autólogo de conjuntiva em ambos e um paciente com ceratoconjuntivite atópica (2,8\%).

Quanto ao uso de esteróides tópicos, 26,2\% dos 172 pacientes analisados utilizaram-nos $(n=45)$. Os pacientes com
CCP foram os que mais usaram estes colírios, com 36,8\% $(n=35)$, seguida pela CCA, com $22,9 \%(n=8)$, e pela CAS, com $6,2 \%$ $(n=2)$, com diferença estatisticamente significativa $(\mathrm{p}<0,03)$. Pacientes com CAP não fizeram uso desta droga. (Gráfico IV)

Quarenta e cinco pacientes usaram corticosteróides tópicos, dos quais 11 pacientes $(24,5 \%)$ usaram a rimexolona; 16 pacientes $(35,5 \%)$, o fosfato de dexametasona $0,1 \%$ e 18 $(40,0 \%)$, o acetato de prednisolona $1 \%$. A análise estatística mostrou tendência a favor de uma diferença significativa.

Na CCP, 35 pacientes (36,8\%) usaram corticosteróides; na CCA, $8(22,9 \%)$; na CAS, $2(6,3 \%)$ e na CAP não houve uso destas medicações.

O tempo de uso de corticosteróides por crise variou de 7 a 60 dias. A freqüência de uso dos esteróides variou de 1 a 4 vezes ao ano. Pacientes com CCP usaram em sua maioria $1 \mathrm{vez}$ ao ano $(\mathrm{n}=20 ; 57,1 \%)$ com média de $0,62 \pm 0,99$, porém 2 pacientes usaram 4 vezes num ano $(5,8 \%)$. Por outro lado, 4 dos 8 pacientes com CCA que usaram (50\%), precisaram uma vez no ano, e os outros 7, 2 vezes no ano, com média de 0,25 $\pm 0,50$. Todos os pacientes com CAS que instilaram esteróides durante o tratamento o fizeram apenas uma vez por ano (média de $0,06 \pm 0,24)$. Estas diferenças foram estatisticamente significativas, com $\mathrm{F}=6,8$ e $\mathrm{p}<0,001$.

\section{DISCUSSÃO}

Nos limites de nosso conhecimento, trata-se do maior estudo sobre conjuntivites alérgicas feito até hoje na literatura nacional, com 172 pacientes portadores de alergia ocular crônica acompanhados por, pelo menos, seis meses em nosso Ambulatório.

Muitas variáveis foram estudadas. A freqüência de aparecimento dos subtipos de conjuntivite alérgica foi muito destoante, já que $55,2 \%$ da amostra foram pacientes com $\mathrm{CCP}$, $20,3 \%$, CCA, 18,6\%, CAS e 5,9\%, CAP. Estes dados refletem a realidade de nosso serviço, que recebe pacientes com alergia ocular severa. Belfort e cols ${ }^{6}$ encontraram valores um pouco diferentes, mas em seu estudo a CCP também foi mais comum, seguida pela CCA. Tal concordância se deve provavelmente por se tratar de pacientes vindos de dois serviços referência 
em um país tropical. Não encontramos os mesmos valores em países com clima temperado, tampouco em estatísticas de clínicas particulares ou de menor porte.

A idade dos pacientes estudados teve média geral baixa, de 11,4 anos, variando de 1 a 48 anos, o que se deve à baixa idade dos pacientes com CCP, cuja média foi a menor entre os 4 subtipos, 9, 1 anos, de 1 a 23 anos, o que se deu por ser a CCP uma forma de alergia ocular que ocorre em crianças e se resolve espontaneamente na adolescência. Estes dados estão de acordo com aqueles encontrados por outros autores ${ }^{(3-4,6,12)}$.

A acuidade visual é um dado importante a ser analisado em pacientes com alergia ocular, principalmente as formas mais severas, como a CCA e a CCP, que cursam com ceratite, e não raramente úlcera em escudo. Estes pacientes muitas vezes têm que fazer uso de corticosteróides para haver controle do quadro, e seus efeitos colaterais muitas vezes causam baixa visão.

Como esperávamos, a CAP não causou diminuição visual, já que pode acometer a córnea; $75 \%$ dos pacientes com CAS obteve visão 1,0 com correção óptica, no melhor olho; a CCA e a $\mathrm{CCP}$, devido às alterações corneais e possíveis seqüelas, como leucoma residual após úlcera em escudo e ceratocone, apresentaram menos pacientes com 1,0 de visão. Por outro lado, a CCA apresentou mais pacientes com visão menor ou igual a 0,5 em relação a CCP, provavelmente pelas alterações ocorridas a longo prazo, já que esta forma de alergia não tem cura ${ }^{(3)}$.

Constatamos que a freqüência das crises segundo a variação climática é realmente acentuada. A CCP mostrou 96,8\% das crises nas épocas do calor, ao passo que $91,4 \%$ dos pacientes com CCA referiu crises no frio. A CAS mostrou piora dos sintomas no verão. Estes dados estão de acordo com a literatura ${ }^{(3,10,12)}$.

Prevaleceu o sexo masculino, com $68,1 \%$ da amostra. A CCP apresentou $67,4 \%$ e a CCA, $60 \%$, diferentemente de Belfort e cols, que encontraram incidência igual entre os sexos para $\mathrm{CCA}^{(5-6)}$.

Quanto ao número de crises por ano, também a CCP apresentou-se mais freqüente, com média superior às outras alergias. Tal fato se deve ao quadro clínico severo e, muitas vezes, de difícil controle.

As alterações corneais são muito comuns nas formas primaveril e atópica ${ }^{(4,13)}$. A ceratite é a mais comum, acometendo $60 \%$ dos pacientes com CCP e 37,2\% dos com CCA. Isto se deve ao quadro inflamatório severo, com liberação de mediadores inflamatórios que agem sobre a córnea, e, na CCP, também ao fator mecânico das papilas gigantes em atrito com a superfície ocular ${ }^{(4,13)}$.

A úlcera em escudo ocorreu em $14,7 \%$ dos pacientes com CCP e 8,6\% dos com CCA, pela mesma fisiopatologia descrita para a ceratite. A CCA não cursa com papilas gigantes, mas as úlceras podem ter ocorrido apenas pelo fator imunológico, com a liberação de enzimas epiteliotoxicas ${ }^{(3-4,13)}$.

Ceratocone é uma ectasia corneal que pode aparecer em indivíduos com alergia. É descrita em pacientes com a forma atópica da doença, sua etiologia ainda não é bem estabelecida, mas pode haver um componente genético e o fato de o pacien- te coçar os olhos pode promover seu desenvolvimento ${ }^{(12)}$. Pacientes com CCA, CCP e CAS, em 14,2\%, 6,4\% e 3,1\% respectivamente, se apresentaram com a doença após o diagnóstico da alergia ocular.

O tratamento das conjuntivites alérgicas com estabilizadores de membrana de mastócito se mostrou eficaz em nossos pacientes. Controlou o quadro em 50,5\% das CCP, 57, $1 \%$ das CCA, $28,5 \%$ das CAS e $80 \%$ das CAP. Apenas na CCP a lodoxamida se mostrou superior a cromoglicato de sódio $4 \%$. Estes dados não estão de acordo com o esperado para $\mathrm{CCP}$, pois devido à severidade do quadro, pensávamos ser pequeno o número de indivíduos controlados com uma droga que não fosse corticóide. Os dados da literatura reforçam a idéia de que pacientes graves necessitam da terapia com esteróides ${ }^{(1,4,7,13)}$.

Muitos pacientes usaram corticóides tópicos em nosso estudo. A maior parte, com diagnóstico de $\mathrm{CCP}$, como se esperava. Porém, não eram a maioria, mas $\operatorname{sim} 36,8 \%$; para CCA, este número caiu para $22,9 \%$, o que reforça, mais uma vez, a idéia de que esta medicação não deva ser usada, a não ser em casos de ceratite punctata superficial, úlcera em escudo ou quadros refratários ao tratamento com estabilizadores de membrana de mastócitos ou medicações de ação combinada ${ }^{(3)}$.

Quarenta por cento dos pacientes tratados com esteróides usaram acetato de prednisolona $1 \% ; 35, \%$ por cento, fosfato de dexametasona $0,1 \%$ e $24,5 \%$, rimexolona, sempre em esquema de pulsoterapia. A freqüência do uso destas drogas foi uma vez ao ano em 57,1\% das CCP, 50,0\% das CCA e 100,0\% das CAS. Pacientes com CCP chegaram a usar até 4 vezes ao ano, por até 60 dias seguidos, reforçando, assim, a idéia de que esta forma de alergia é muito severa ${ }^{(1,4,7,13)}$.

Pacientes com CCP relativamente controlada, olho calmo, porém com ceratite intensa ou úlcera em escudo são beneficiados com a ressecção de papilas gigantes. Em nosso serviço, utilizamos a técnica proposta por Nishiwaki-Dantas e cols, com transplante livre de conjuntiva autóloga. Em nosso estudo, $7,4 \%$ das CCP e 2,8\% das CCA foram submetidos a este procedimento, evoluindo, a partir daí, para melhora do quadro corneal.

\section{CONCLUSÕES}

A CCP é o tipo de conjuntivite alérgica mais freqüente em nosso serviço, o que provavelmente se deve não somente ao clima tropical, mas também ao fato de ser este um hospital de referência para os casos graves desta enfermidade. A droga mais eficaz na nossa experiência é o cromoglicato dissódico, muito usado em nosso ambulatório devido ao baixo custo e aos resultados confiáveis a longo prazo.

Corticosteróides são potentes agentes antiinflamatórios que, nestes pacientes, muitas vezes são as únicas drogas capazes de cessar as crises e evitar complicações corneais permanentes com baixa de visão definitiva. 


\section{ABSTRACT}

Purpose: To establish a profile of the allergic patient based on clinical manifestations, epidemiological data, treatment response and complications of ocular allergy. Methods: A retrospective and descriptive study, in which we analyzed data of 172 patients suffering from perennial conjunctivitis, seasonal conjunctivitis, atopic keratoconjunctivitis and vernal keratoconjunctivitis, with at least 6 months of follow-up. The study was performed at the Ocular Allergy Sector of the "Santa Casa de São Paulo". Statistical analysis was performed using variance method and qui-square test. Results: The most frequent form of ocular allergy was vernal keratoconjunctivitis $(\mathrm{n}=95 ; 55.2 \%)$ and the male sex was predominant $(\mathrm{n}=117$; $68.1 \%)$. Mean age was 111.7 years $( \pm 8.7)$. Vernal keratoconjunctivitis was the disease that most impaired vision, also responsible for corneal complications in $60 \%$ of the patients $(\mathrm{n}=57)$. Among medications, corticosteroids were used by $21.6 \%(\mathrm{n}=45)$ of patients and $36.8 \%$ of them had vernal keratoconjunctivitis. Disodium cromoglycate was not only the most used medication, but able to successfully control symptoms in most cases. Papilla resection with autologous transplantation had to be performed in 8 patients. Conclusions: Vernal keratoconjunctivitis showed to be the most frequent form of ocular allergy in our service. The most efficient drug in our experience seemed to be disodium cromoglycate. Corticosteroids were shown to be as potent anti-inflammatory drugs and sometimes were the only way to discontinue crises.

Keywords: Conjunctivitis, allergic/epidemiology; Conjunctivitis, allergic/complications; Conjunctivitis, allergic/drug thera- py; Keratoconjunctivitis/drug therapy; disodium cromoglycate/therapeutic use; disodium cromoglycate/administration \& dosage

\section{REFERÊNCIAS}

1. Abelson MB, Schaefer K. Conjunctivitis of allergic origin:imunologic mechanisms and current aproaches to therapy. Surv Ophthalmol 1993;38 (Suppl):115-31.

2. Titi MJ. A critical look at ocular allergy drugs. Am Fam Phys 1996;53:2637-42.

3. Nishiwaki - Dantas MC. Conjunvite alérgica. In: Conselho Brasileiro de Oftalmologia. Manual do CBO: 1999. Rio de Janeiro: Cultura Médica; 2000. p.210-21.

4. Abelson MB, George MA, Garofalo C. Diferential diagnosis of ocular allergic disorders. Ann Allergy 1983;70:95-113.

5. Bonini S, Bonini S, Lambiase A, Marchi S, Pasqualetti P, Zuccaro O, et al. Vernal keratoconjunctivitis revisited. A case series of 195 patients with longterm followup. Ophthalmology 2000;107:1157-63.

6. Belfort R, Marback P, Hsu C, Freitas D. Epidemiological study of 134 subjects with allergic conjunctivitis. Acta Ophthalmol Scand 2000;78 (Suppl 230):38-40.

7. Tabbara KF. Ocular complications of vernal keratoconjunctivitis. Can J Ophthalmol 1999;34:88-92.

8. Maichuk IF. New aspects in drug therapy of ocular allergies. Vestn Oftalmol 2000;116:10-4.

9. Leonardi A. Role of histamine in allergic conjunctivitis.Acta Ophthalmol Scand 2000;78(Suppl 230):18-21.

10. Nishiwaki- Dantas MC, Dantas PE, Pezzutti S, Finzi S.Surgical resection of giant papillae and autologus conjunctival graft in patients with severe vernal conjunctivitis and giant papillae. Ophthalmic Plast Reconstr Surg 2000;16: 438-42.

11. Rapoza PA, Quinn TC, Terry AC, Gottsch JD, Kiessling LA,Taylor HR. A sistematic approach to the diagnosis and treatment of chronic conjunctivitis. Am J Ophthalmol 1990;109:138-42.

12. Friedlaender MH. Allergic conjunctivitis. In: Krachmer JH, Mannis MJ, Holland EJ, Krachmer P. Cornea. Fundamentals of cornea and external disease. St. Louis: Mosby; 1997. p.805-10.

13. Friedlaender MH. Management of ocular allergy. Ann Allergy Asthma Imunolol 1995;75:212-22.

\section{Congresso Norte-Nordeste de Offalmologia}

\section{0 de junho a 3 de julho de 2.004 Teresina - PI}

INFORMAÇÕES: Tel./Fax: (86) 223-9444

E-mail: oftalmologia.nortenordeste.2004@bol.com.br 